\title{
EVALUATION METRICS APPLIED TO ACCIDENT TOLERANT FUEL CLADDING CONCEPTS FOR VVER REACTORS
}

\author{
Martin ŠEveČEK ${ }^{a, b, *}$, MoJmír VALACH $^{c}$ \\ ${ }^{a}$ Department of Nuclear Reactors, Faculty of Nuclear Sciences and Physical Engineering, Czech Technical \\ University in Prague, V Holešovičkách 2, Praha 8, Czech Republic \\ b ALVEL a.s., Opletalova 37, Praha 1, Czech Republic \\ ${ }^{c}$ UJV Řež, a.s., Department of Severe Accidents and Thermomechanics, Nuclear Power and Safety Division, \\ Hlavni 130, Řě,, 25068 Husinec, Czech Republic \\ * corresponding author: martin.sevecek@fji.cvut.cz
}

\begin{abstract}
Enhancing the accident tolerance of LWRs became a topic of high interest in many countries after the accidents at Fukushima-Daiichi. Fuel systems that can tolerate a severe accident for a longer time period are referred as Accident Tolerant Fuels (ATF). Development of a new ATF fuel system requires evaluation, characterization and prioritization since many concepts have been investigated during the first development phase. For that reason, evaluation metrics have to be defined, constraints and attributes of each ATF concept have to be studied and finally rating of concepts presented. This paper summarizes evaluation metrics for ATF cladding with a focus on VVER reactor types. Fundamental attributes and evaluation baseline was defined together with illustrative scenarios of severe accidents for modeling purposes and differences between PWR design and VVER design.
\end{abstract}

KEYWORDS: nuclear fuel, accident tolerant fuel, evaluation metrics, LWR, VVER.

\section{INTRODUCTION}

After the events at the Fukushima Daiichi NPP, enhancing the accident tolerance of LWRs became a topic of high interest in many countries. The goal of accident tolerant fuel (ATF) development is to develop alternative fuels to further enhance the safety, competitiveness, and economics of nuclear power. The world's leader in ATF development are the U.S., where in 2012, the Congress directed to DOE to:

- Develop enhanced fuels and cladding for LWRs to improve safety in the event of accidents in the reactor or spent fuel pools.

- Emphasize and fund activities aimed at the development and near-term qualification of meltdownresistant ATF that would enhance the safety of present and future generations of LWRs.

- Create a plan for development of meltdown-resistant fuels leading to reactor testing and utilization by 2020.

Other countries (e.g. France, South Korea, China) have been conducting their own ATF research with similar goals. Recently, Russia introduced its plan for ATF development with a focus on VVER reactors. Activities related to the ATF development are also coordinated by international organizations as IAEA or OECD/NEA. The initial effort is focused on applications in operating LWR reactors (PWR, BWR, and VVER). The goal set by the U.S. or South Korea is to insert a lead test assembly (LTA) into a commercial reactor by 2022. Other countries including Russia or the Czech Republic have not defined their specific goals, yet.
This paper summarizes an evaluation methodology, proposed set of metrics, related tests and illustrative accident scenarios for the evaluation of VVER ATF cladding concepts. The metrics are based on the detailed evaluation approach proposed in the [1, with an emphasis given on the fuel cladding, VVER reactors, and their departures from standard PWR reactor design. The evaluation methodology will be applied to assess each ATF cladding concept relative to the current VVER fuel system and will be described in details in future technical report.

\subsection{ATF Definition}

ATF fuels are according to the OECD/NEA [2] defined as fuels that can tolerate a severe accident in the reactor core for a considerably longer time period than the current $\mathrm{UO}_{2}-\mathrm{Zr}$ alloy fuel system, while maintaining or improving the fuel performance during normal operations and operational transients.

\subsection{Coping Time}

Each concept will be evaluated besides other characteristics based on its ability to increase the "coping time" under severe accident conditions. Where coping time is according to the OECD/NEA defined as 2]: the time to significant loss of geometry such that the fuel can no longer be cooled or cannot be removed from the reactor. For each concept, there will be an analysis of failure modes and effects (FMEA) performed to determine the onset of particular initiating event that leads to severe accident. However, the common baseline has to be set and general illustrative scenarios defined. 


\subsection{Development Strategy}

The strategy for ATF development includes three phases:

(1.) Feasibility assessment and down-selection (laboratory experiments; code updates; assessment of economical, operational, safety, fuel cycle, and environmental impacts using evaluation metrics).

(2.) Development and qualification (ATF fabrication; irradiation; safety basis testing; qualification and licensing).

(3.) Commercialization (technology transfer to industry).

Any new ATF concept must comply with current safety and performance constraints, fuel cycle impacts or additional LWR design constraints. Moreover, it's attributes should be better in comparison with the current fuel system to achieve comparable or better performance in normal and accident conditions. For that reason, the quantitative metrics have to be developed and described in details taking into account also the requirements of utilities, fuel vendors, and regulatory bodies.

\subsubsection{Challenges in Developing ATF}

One of the main concerns is a definition of testing and qualification requirements. Individual attributes are not equal, which requires concepts prioritization and determination which attributes to test and what metrics are needed to evaluate attribute compliance.

If new ATF concept should be accepted by utilities and vendors, it must be capable of integration into the current nuclear fuel cycle system (Figure 1). The challenge is to get the best performance at each step of nuclear fuel cycle, and to understand how it affects other parts of the system.

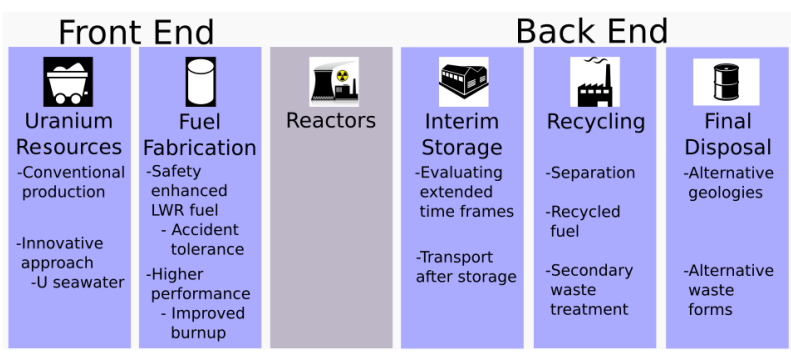

FIGURE 1. ATF shall not negatively (economically or technically) impact fuel cycle technologies. For that reason, integrated evaluation approach has to be adopted.

\section{Attributes of ATF}

The attributes for mitigation of fuel failure during severe accidents provide fundamental guidance for ATF evaluation. It may not be necessary to improve fuel system in all attributes. Some attributes may provide substantial enhancement in accident tolerance, while others may provide only marginal benefits. The desired attributes highlight the performance of the fuel under normal and accident conditions. Key attributes for a fuel system demonstrating enhanced accident tolerance include reduced steam reaction kinetics, lower hydrogen generation rate, and reduction of the initial stored energy in the core. The desired behaviors should be accomplished while maintaining or improving cladding thermo-mechanical properties, fuel-cladding interactions, and fission-product retention.

In some cases, the described consequences of accident conditions may be concept-specific (e.g. hydrogen generation in the current $\mathrm{Zr}$ alloy- $\mathrm{UO}_{2}$ fuel system). Other ATF concepts could present additional effects not expressed here (e.g. generation of CO in case of $\mathrm{SiC}$ ) that must be considered in evaluation of the proposed system. A brief description of the desired attributes is provided in this section and summarized in Figure 2

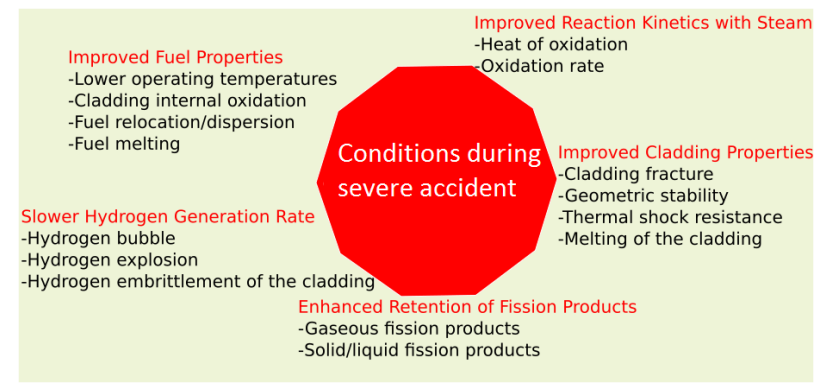

Figure 2. Major issues that need to be addressed in establishing accident tolerant fuel attributes.

Candidate fuel systems must first NOT HARM, which means that the fuels must perform as well as or better than the current fuel system. Moreover, the fuel system must additionally provide a comparable or improved response to AOOs (Anticipational Operational Occurences), DBAs (Design Basis Accidents) and BDBAs (Beyond Design Basis Accident).

\subsection{Hydrogen Generation Rate}

Hydrogen generation in the reactor core can lead to energetic explosions similar as in Fukushima-Daiichi accident. Under a high-temperature steam environment, it is not possible to avoid hydrogen generation with standard Zr-alloys. Rapid oxidation of cladding results in free hydrogen generation. This exothermic reaction increases the cladding temperature, which further accelerates free hydrogen generation. A related issue is the diffusion of free hydrogen into the unoxidized portion of the cladding, resulting in enhanced embrittlement and potential cladding failure.

The desired alternative is a cladding material that resists oxidation or reduces the rate of oxidation resulting in a slower hydrogen generation rate. Materials with lower heat of oxidation are important due to the limitation of temperatures during an accident. Materials that are less susceptible to hydrogen diffusion 
may address the rapid embrittlement issue typical for standard Zr alloys.

\subsection{Fission Product Retention}

Cladding provides an important barrier between fission products and primary circuit. The potential release of fission products to the environment has to be avoided, therefore, retention within the fuel is of the highest importance. While total retention may not be possible, higher partial retention would be a substantial improvement.

The desired improvement is to prevent melting or dispersion of the fuel by utilization of hightemperature/strength cladding materials that would retain cladding integrity beyond the current limitations of Zr-alloy cladding.

\subsection{Cladding Reaction with Steam}

When cladding is exposed to steam at high temperature, multiple issues need to be considered: hightemperature steam interaction with $\mathrm{Zr}$-alloy cladding, an exothermic oxidation reaction, hydrogen generation, and degeneration of the structural integrity of the cladding.

ATF materials should demonstrate enhanced tolerance to radiation and oxidation under hightemperature exposure while specifically considering mechanical strength and structural integrity at the end of life and when exposed to high-temperature steam for an extended duration.

\subsection{Fuel-Cladding Interactions}

Pellet cladding chemical interactions (PCCI), pellet cladding mechanical interactions (PCMI) and fuel heating are important properties that must be understood during normal operation and accident conditions for all new fuel concepts.

The desired design option is to develop fuels with reduced PCCI and PCMI, with lower operating temperatures relative to the $\mathrm{Zr}$ alloy- $\mathrm{UO}_{2}$ system, with higher melting point, and with structural integrity at high temperatures. Chemical and physical compatibility of cladding and fuel for all proposed concepts must be ensured.

\section{Constraints on Development OF ENHANCED ATF}

Except for a few rare events, the current $\mathrm{UO}_{2}-\mathrm{Zr}$ fuel system meets all performance and safety requirements while keeping nuclear energy economically competitive. Any new ATF concept should be compliant with and evaluated against current design, operational, economic, and safety requirements. Fuel cycle considerations must also be considered, especially for concepts that represent a significant departure from the current technology. A brief summary of the constraints is illustrated in Figure 3 .

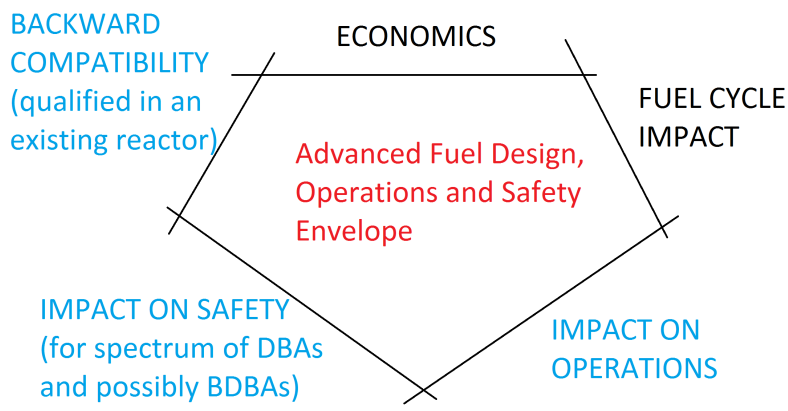

Figure 3. Constraints on New Fuel Designs.

The main constraints include:

- Potential ATF geometry deviations and compatibility with co-resident fuel.

- Evaluation of all potential accident condition parameters, including:

$\triangleright$ FMEA completion to ensure that any potential ATF operating vulnerabilities are recognized and mitigated as possible.

$\triangleright$ Completion of a $\S 50.59$-like process (Changes, tests and experiments section from the 10CFR50 regulation) to illuminate any necessary licensing or logistical preparations for operation of the ATF in a commercial LWR. The process and its guidance is described in 3 .

- Quantifying the recommended minimum additional coping time to be provided by the ATF.

- The need to include a metric to address those concepts that require enrichment greater than $5 \mathrm{wt} \%$.

- The mission of the utility is not to test new fuels. Any ATF operation must necessarily address and minimize impacts on the utility by ensuring full compatibility with co-resident fuel and reactor components, and by limiting perturbations of the normal operation of the plant.

- Nuclear industry has optimized the current $\mathrm{Zr}-\mathrm{UO}_{2}$ which represents a large financial investment. The additional cost of ATF must be relatively low. At present, it is not feasible to determine quantitative results.

\subsection{Backward Compatibility}

ATF concepts must be suitable for use in existing LWRs. Longer term concepts may be considered in conjunction with the near-term focus. Proposed fuel concepts should not require plant modifications to the current reactors. They should be compatible with existing fuel handling equipment, fuel rod or assembly geometry, and co-resident fuel in existing LWRs.

\subsection{IMPACT ON OPERATIONS}

A concept must maintain or extend plant operating cycles, reactor power output, and reactor control. Re- 


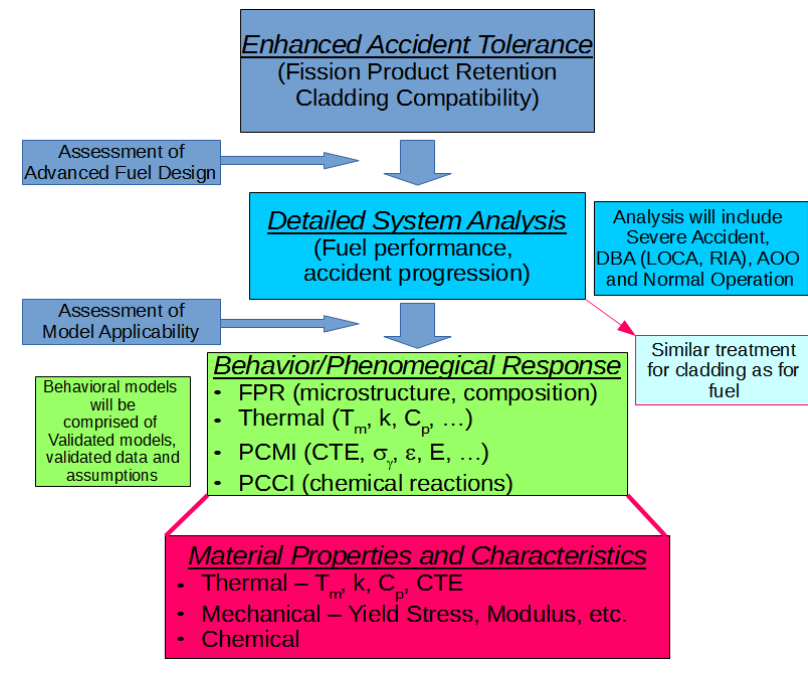

Figure 4. A diagram that describes an approach to assessing enhanced accident attributes of a proposed fuel design.

ducing the availability or power output would be unacceptable to utilities. To maintain current operation, some of the fuel system concepts would require higher fuel enrichment. While the impact of higher enrichment is fairly well understood from a technical perspective, regulatory and safeguard issues have to be addressed.

\subsection{ECONOMIC IMPACTS}

Any ATF concept is likely to be more expensive than the current $\mathrm{UO}_{2}-\mathrm{Zr}$ system, at least initially. Fuels that require higher enrichment are especially likely to cost more. Increased enrichment could additionally require modifications to fuel fabrication facilities. Therefore, it is important to carefully assess the economic impact of the new technology and to determine how much additional fuel cost the utilities will accept. On the other hand, increasing burnup and power densities (power upgrades) could reduce or mitigate the negative economical impacts.

\subsection{SAFETy IMPACT}

Performance of a new fuel system will be compared to the performance of the $\mathrm{UO}_{2}-\mathrm{Zr}$ alloy system which defines a baseline for the ATF evaluation. The performance must be shown to be similar to or better than of the current system.

\subsection{Fuel Cycle Impact}

ATF must adhere to regulations and policies, for both the fuel fabrication facility and the operating plant, with respect to technical, regulatory, equipment, and fuel performance considerations. A new ATF system could also have an impact on the back-end of the fuel cycle. The storage, repository performance of the fuel or possibility of reprocessing must not be degraded.

\section{Metrics and Related Testing}

The metrics determine a clear technical methodology for evaluation that can be used to rank two or more concepts. The approach has to be simplified due to the complex multiphysics behavior of nuclear fuel and the large set of performance requirements that must be met. Dozens types of ATF cladding ranging from metallic to fully ceramic cladding each with very different material properties and behavior has been studied. Each concept has to be evaluated and compared to determine the concept viability, and prioritize resources to obtain the cladding with the best compromise in terms of properties and behavior in both nominal and accidental conditions.

\subsection{Desired cladding properties, BEHAVIOR AND PERFORMANCE}

The cladding of a nuclear fuel has three fundamental functions:

(1.) Confinement of fissile material and fission products within the fuel rod.

(2.) Not affecting chain reaction (low absorption cross section).

(3.) Efficient heat transfer from the fuel to the coolant.

To fulfill these three functions a new ideal cladding material needs to have certain properties and exhibit specific behaviors as follows:

Fabricability:

- Compatibility with fabrication facilities; Material availability; Welding/sealing behavior.

Thermo-mechanical behavior:

- High thermal conductivity, strength, and ductility; Leak-tightness throughout plant operation (impermeable to fission gas and fission products).

Corrosion behavior:

- High corrosion resistance in VVER reactor environment; High corrosion resistance in high temperature steam; Low $\mathrm{H}_{2}$ production and low hydrogen embrittlement.

Chemical compatibility:

- Compatibility with fuel, coolant, and the fuel assembly components; No eutectic.

Irradiation behavior:

- Stable or predictable thermal, mechanical, and corrosion behavior under irradiation; Dimensional stability; Low irradiation embrittlement; Low thermal neutron absorption cross-section; Low activation.

Back-end behavior:

- Low tritium permeation; No impact on the reprocessing; Satisfactory storage behavior.

New ATF cladding material will not exhibit all presented ideal properties. For that reason, a baseline for evaluation and assessment of various concepts has 
to be established. This corresponds to the metrics described further.

\subsection{Metrics Definition}

The metrics are divided into three sections:

(1.) Material properties inherent to the material.

(2.) Material behavior observed through standardized tests or experiments for normal and accident conditions.

(3.) Material behavior observed through standardized tests for accident conditions.

Note that, material behavior can depend also on particular reactor type and its performance.

\subsubsection{Inherent Material Properties}

The inherent material properties that should be compared are mainly thermal and neutronic properties: High Melting/Sublimation temperature; High thermal conductivity; Optimal heat capacity/enthalpy; Optimal thermal expansion coefficient to ensure dimensional stability; Thermal neutron absorption crosssection.

There are other concept-specific inherent properties. For example in case of multi-layer coating, compatibility between different materials has to be ensured. Additionally, eutectic temperature might decrease the melting temperature of the cladding so it should be avoided.

\subsubsection{Normal Operation Behavior}

In contrast with the inherent physical properties described above, the behavior of the materials has to be assessed through tests with standardized conditions to enable direct comparison and evaluation of the various concepts.

Long-term corrosion tests are proposed to evaluate the corrosion behavior in normal operating conditions in a different chemical environment. An acceleration of kinetics is likely under irradiation which has to be also investigated. The important data to assess are: Visual inspection - Potential delamination of coatings; Corrosion kinetics - Weight change, Oxide thickness; Water chemistry analyzes; Hydrogen pick-up.

Mechanical tests suggested for mechanical behavior evaluation are: Tensile tests; Internal pressure creep tests; Internal pressure burst tests; Leaktightness behavior; Fatigue tests - Thermal cycling, Internal pressure load cycling.

\subsubsection{Accident Conditions Behavior}

First, tests simulating normal operating conditions will be performed. Later, the material performance under selected accident conditions will be assessed. Specific tests selected to determine accident performance of candidate cladding materials are series of standard tests simulating RIA and LOCA conditions.

For example: High temperature oxidation, Isothermal internal pressure burst tests at multiple temperature set points, Dynamic internal pressure burst tests with increasing temperature until cladding failure, Rapid burst test with increasing temperature until cladding failure, Tensile tests at elevated temperature, internal burst tests, tensile test at elevated temperature etc.

\section{Evaluation Baseline}

At the beginning of the evaluation, it is recommended to establish a common understanding of the current state-of-the-art cladding. Key characteristics and performance criteria were identified, with quantitative values assigned where possible. All of the parameters are $\mathrm{Zr}$ alloy specific, some are reactor design and reactor performance (chemistry regime, power changes) specific. However, there can be common baseline for most of LWRs defined with only small uncertainties.

\subsection{Baseline for Normal Operating Conditions}

Generally accepted characteristics of Zr-based alloys during normal operations are 4]:

- Fission product retention: $10^{-6}$ pin failure rate, Radiation tolerant: up to $10 \mathrm{dpa}, 10^{22} \mathrm{n} / \mathrm{cm}^{2}$ fast $(>1 \mathrm{MeV})$, Dimensional stability: $\max 1 \%$ axial elongation.

- Thermal conductivity $\lambda: \sim 17.4 \mathrm{~W} / \mathrm{mK}$, Heat capacity $C_{p}: 293 \mathrm{~J} / \mathrm{kg} \mathrm{K}$, Heat transfer coefficients: coolant/cladding coupling (reactor design dependent).

- Corrosion performance: Oxide growth $\leq 100 \mu \mathrm{m}$, Hydrogen content $\leq 800$ wppm, Maximum limit on corrosion product buildup (linked to water chemistry specifications), Galvanic corrosion limits, Requirement for all corrosion products to be harmless to other system components.

- Tritium release into coolant: Limit for tritium permeation not established, but "low" permeation is desirable, $<10 \%$ of the total tritium generated permeating into the coolant could be reasonable. Vendors assume that less than $2 \%$ of this tritium permeates through Zr-based alloy cladding to the coolant (e.g. for SS-316, the permeation could be as high as $90 \%)$.

- Hydrogen pick-up: Typical for Zr-based alloys, Depends on corrosion level and varies with alloy from $100 \mathrm{ppm}$ to $800 \mathrm{ppm}$ (600 ppm should be acceptable under normal operating conditions).

- Mechanical criteria - Ductility: Currently $1 \%$ minimum of total elongation; modern alloys show uniform elongation up to $5 \%$ and total elongation up to $20 \%$; Yield strength: current as-fabricated alloys, 150-400 MPa at $340^{\circ} \mathrm{C}$, Ultimate tensile strength: $437 \mathrm{MPa}$ at room temperature (Zry-4), Creep strain limit: $0.66 \%$ (Zry-4); Resistance to crack propagation (fracture toughness). 
- Fretting wear: $<10 \%$ of wall thickness; Debris wear resistance; Fabricable: weldable, thin-walled tube that maintains hermeticity.

- PCI interaction behavior: Includes consideration of fission product interactions (possible SCC), Oxide layer on the inside of the clad ( $\sim 10$ micron).

- Burnup limit: peak rod average: $62 \mathrm{MWd} / \mathrm{kg} \mathrm{U}$.

\subsection{Baseline for Accident Conditions}

The accident performance of Zr-based cladding differs from the normal operation behavior. The main current metric for fuel system performance is that the cladding should maintain post-quench ductility by limiting the peak cladding temperature to $1204^{\circ} \mathrm{C}$ and the maximum oxidation level to $17 \%$ of the cladding wall thickness. It has been shown that the temperature and oxidation limits result in maintaining ductility for as-fabricated and very low burnup cladding. Changes underway NRC Regulatory Guides and IAEA guidelines will limit the oxidation level for DBAs based on pre-DBA hydrogen content (e.g. $4 \%$ for $600 \mathrm{wppm}$ hydrogen content) [5].

Under beyond BDBA LOCA conditions, cladding will be subjected to higher temperatures and oxidation levels. Zr-based cladding alloys would experience longer times at increasing temperatures, higher oxidation rates, higher hydrogen release rates, and higher internal heat-of-oxidation rates. Key performance measures for fuel behavior during accident conditions include coping time, behavior under elevated temperature conditions over long periods of time, and material oxidation [6]. The relevant temperatures for LWR severe accidents which result in the formation of liquid phases due to melting or chemical interactions are summarized in Figure 5 .

- Coping time under DBA and BDBA - LBLOCA conditions: from 50 to $300 \mathrm{sec}$ at $1204^{\circ} \mathrm{C}$, SBLOCA conditions: $\sim 1 \mathrm{hr}$ at $\leq 1050^{\circ} \mathrm{C}$.

- Elevated temperature issues - Melting temperature: $\sim 1760{ }^{\circ} \mathrm{C}$, Temperature for eutectic formation (decrease of melting point, effects on other components), Runaway oxidation - relates to heat of oxidation and acceleration of the oxidation process.

- Steam oxidation kinetics: Oxidation rate and associated heat of oxidation, Performance considered for time at elevated temperature and maximum temperature, Breakaway oxidation may occur at elevated temperature (e.g. time at $\sim 1000^{\circ} \mathrm{C}$ before breakaway oxidation occurs should be defined).

- Hydrogen production and release: $<1 \% \mathrm{Zr}$-metal conversion.

- Maintain ductility following DBA: Most important accident performance criterion under licensing standards, Regulation specifies at least $1 \%$ ductility post-quench.
- High temperature mechanical properties: Balloon and burst are not currently defined as a fuel failure and are not limited in the licensing criteria; While only a fraction of rods burst, those that do typically burst at $\sim 800^{\circ} \mathrm{C}$; Burst may occur at lower temperature with higher burnup due to higher internal pressure; Burst is a ductile failure; Size of ballooning and burst opening can be important to the results of the failure.

- Severing and/or shattering the cladding with subsequent fuel release are considered as a failure; Creep performance must be also considered.

- Flow blockage under accident scenarios: Required calculation for licensing, Related to maintaining coolable geometry, Flow blockage may result from swelling / ballooning of cladding.

- Thermal shock resistance: Current cladding alloys do not shatter until they have reached high oxidation levels $(>17 \%$ of the cladding wall thickness consumed by oxidation), Standard test applied to determine thermal shock performance.

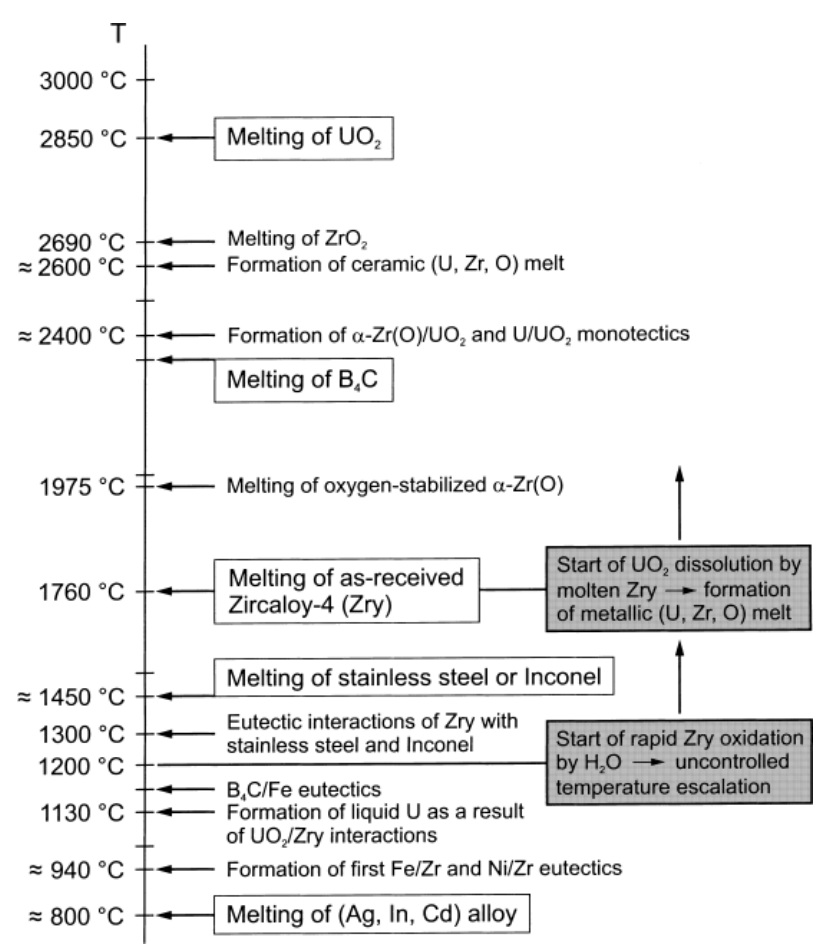

FiGURE 5. LWR severe accident-relevant melting and chemical interaction temperatures which result in the formation of liquid phases 7 .

The goals of evaluation are associated with defining requirements for down-selection among options during the feasibility assessment. It includes identification of important attributes for ATF, understanding the common baseline for evaluation and map the merit of the attributes against potential operational or safety envelope benefits which are presented in the previous sections. It is also required to define safety analyzes including accident scenarios to quantify the target 
values of particular ATF fuel characteristics which are presented in the next section.

\section{Illustrative Scenarios for ATF EVALUATION}

Two "bounding scenarios" for BDBA evaluation are proposed. They cover wide range of severe accidents and at the same time are not too prescriptive and specific to a particular NPP design. Modeling of these scenarios will utilize one of the initiating events and will continue until a defined point of failure as defined in by the coping time definition (see Section 1.2 for particular ATF concept.

The two scenarios applicable to VVER reactors are:

- Long-term Station Blackout: high-pressure scenario; it will be calculated to the point of reactor pressure vessel failure.

- Large-break LOCA: low-pressure scenario with high decay power and limited availability of emergency cooling systems.

The proposed scenarios are intended to provide bounding cases for fuel performance. Each ATF concept will be evaluated by fuel performance and system analysis codes in regard to the two illustrative scenarios. All ATF evaluations should be allowed to progress to the point defined by the coping time definition with considering failure modes and effects for particular ATF concept. The goal of the evaluation is to estimate the potential increase in coping time that is offered by the ATF concept and to assess potential outcomes (e.g. fuel failure, coolability, cause of failure). Following completion of bounding (most severe) analyzes, more detailed studies for these illustrative scenarios should be performed to develop a better understanding of the impact of additional variables as burnup, time after SCRAM when core cooling is lost etc.

\subsection{High Pressure Scenario - SBO}

The high-pressure SBO (Station Blackout) scenario for ATF evaluation purposes for VVER-1200 reactor is defined as:

- Loss of all sources of AC supply.

- Feed water supply is unavailable.

- Turbine isolation valve is activated, pressure in the steam generators (SGs) increases.

- After exceeding the pressure limit the quick-acting pressure reducing system opens.

- A failure of the quick-acting pressure reducing system is postulated - system stays open after pressure decreases.

- Loss of coolant in SGs leads to decay heat removal passive system failure.

\subsection{Low Pressure Scenario - LBLOCA $+\mathrm{SBO}$}

The low-pressure scenario for ATF evaluation purposes for VVER reactors is defined as:

- Guillotine rupture of the primary circuit's cold leg near the reactor inlet with SBO.

- Decay heat removal passive system (DHRS) and emergency core cooling system are in operation.

Operation of the Hydro accumulators is assumed. The decay heat removal passive system and emergency core cooling system operate according to their design characteristics: ECCS - up to water volume exhaustion, DHRS - up to the end of nitrogen absorption due to ECCS operation. Total operation time of these systems is approximately $24 \mathrm{~h}$ from the accident start.

\section{VVER Fuel Specifics}

The Russian design pressurized water reactors called VVER have specific differences in comparison with western PWRs. It is recommended to adopt all evaluation metrics with both illustrative scenarios presented above and define additional VVER specific metrics. The main differences of the VVER fuel and primary circuit include:

- different water chemistry,

- hexagonal geometry and arrangement of the fuel assemblies,

- various cladding and fuel materials

$\triangleright$ E110 alloy, E635 alloy,

$\triangleright$ annular pellet, different dopants, only Gd-based burnable absorbers,

- assembly shroud - in case of VVER-440 fuel assemblies,

- different control rod materials.

The main difference between VVERs and PWRs can be observed in nominal operation due to different chemistry of primary circuit and different material composition. While PWRs use $\mathrm{LiOH}$ for chemistry control, VVERs use KOH. This difference in chemistry regime leads to slightly different test conditions for normal operation corrosion tests. However, in accident conditions the performance of VVERs is similar to that of PWRs. Therefore it is not necessary to define additional VVER-specific metrics for accident conditions.

\section{Conclusion}

To develop new accident tolerant fuel systems an evaluation and prioritization of ATF concepts has to be performed at the end of the first development phase. The prioritization will allow researchers and decision makers to focus resources on most promising concepts. Due to the complicated complex multiphysics behavior of nuclear fuel it is not possible to test all the required characteristics for each concept. For that 
reason, evaluation metrics have to be defined. A good starting point for ATF evaluation establishment is a common understanding of the current state-of-the-art Zr-cladding and its performance in BDBA conditions. It is a baseline for evaluation and assessment of various concepts.

Evaluation of ATF cladding must consider the complete fuel system (including fuel cycle) and should encompass all performance regimes for the fuel, including: Fabrication, Normal operation and AOOs, DBAs, BDBAs, and Used fuel storage, transportation, and disposition. There are numerous attributes within each regime that must be considered in evaluating the fuel system performance. Key attributes for the cladding were discussed in Sections 4 and 5, along with a summary of standard tests recommended for measuring specific properties and characterizing performance under the specified conditions. It may not be possible to improve the current state-of-the-art fuel system in all attributes and regimes, significant improvement in some of the key attributes may outweigh modest performance gains or modest vulnerabilities in other attributes.

A detailed list of proposed attributes for evaluation is provided together with metrics and standardized tests. The attributes summarized in this document, can be used as a qualitative guide to assess the performance of candidate materials relative to the current state-of-the-art materials and relative to one another. Evaluation baseline for common understanding of the current cladding was presented. To further determine the common understanding of ATF performance two severe accidents were defined and described. Based on the illustrative scenarios the coping time can be calculated and ATF concepts evaluated. Most of the metrics and attributes are generally applicable for all LWR reactors but some attributes as chemistry regime are reactor-specific and for that reason additional metrics and standardized test were defined for VVER types of reactors. Detailed more prescriptive approach for evaluation of ATF cladding for VVERs will be described in future technical report.

\section{ACKNOWLEDGEMENTS}

This work was supported by the Grant Agency of the Czech Technical University in Prague, grant No. SGS OHK4-008/16.

\section{REFERENCES}

[1] S. M. Bragg-Sitton, J. Carmack, F. Goldner. Evaluation metrics applied to accident tolerant fuels. Tech. rep., Idaho National Laboratory (INL), 2014.

[2] EGATFL. Light water reactor accident tolerant fuel: Evaluation metrics and technology readiness level definition. Tech. rep., OECD/NEA, 2016.

[3] USNRC. 1.187, Regulatory guidance for implementation of 10CFR50.59. Tech. rep., 2000.

[4] M. Billone, Y. Yan, T. Burtseva, et al. Cladding embrittlement during postulated loss-of-coolant accidents. Tech. rep., Argonne National Laboratory (ANL), 2008.

[5] G. Youinou, R. S. Sen. Enhanced accident tolerant fuels for LWRs-a preliminary systems analysis. Tech Rep 2013.

[6] L. Braase. Enhanced accident tolerant LWR fuels national metrics workshop report. Tech. rep., Idaho National Laboratory (INL), 2013.

[7] P. Hofmann. Current knowledge on core degradation phenomena, a review. Journal of nuclear materials 270(1):194-211, 1999. 